\title{
Pharmacokinetics, Pharmacodynamics, and Safety of Canagliflozin in Japanese Patients with Type 2 Diabetes Mellitus
}

\author{
Hiroaki Iijima $\cdot$ Takayuki Kifuji $\cdot$ Nobuko Maruyama $\cdot$ Nobuya Inagaki
}

To view enhanced content go to www.advancesintherapy.com

Received: July 10, 2015 / Published online: August 18, 2015

(C) The Author(s) 2015. This article is published with open access at Springerlink.com

\begin{abstract}
Introduction: Canagliflozin is a sodium glucose co-transporter 2 inhibitor approved worldwide for the treatment of patients with type 2 diabetes mellitus (T2DM). The present study evaluated pharmacokinetics, pharmacodynamics, and safety of canagliflozin in Japanese patients with T2DM.
\end{abstract}

Methods: Canagliflozin, at doses of 25, 100, 200 , or $400 \mathrm{mg}$, was administered as a single

Electronic supplementary material The online version of this article (doi:10.1007/s12325-015-0234-0) contains supplementary material, which is available to authorized users.

H. Iijima $(\bowtie)$

Medical Affairs Department, Mitsubishi Tanabe

Pharma Corporation, Tokyo, Japan

e-mail: Iijima.Hiroaki@mm.mt-pharma.co.jp

T. Kifuji

Development Division, Clinical Pharmacology

Department, Mitsubishi Tanabe Pharma

Corporation, Tokyo, Japan

N. Maruyama

Development Division, Clinical Research

Department II, Mitsubishi Tanabe Pharma

Corporation, Tokyo, Japan

N. Inagaki

Department of Diabetes, Endocrinology and

Nutrition, Kyoto University Graduate School of

Medicine, Kyoto, Japan dose and, after a washout of 1 day, in repeated doses for 14 consecutive days to 61 subjects in a randomized, double-blind, placebo-controlled study. Plasma concentrations of canagliflozin and urinary glucose excretion (UGE) were measured, and renal threshold for glucose excretion $\left(\mathrm{RT}_{\mathrm{G}}\right)$ was calculated. Safety was evaluated on the basis of adverse event (AE) reports, blood and urine laboratory parameters, and vital signs.

Results: Plasma canagliflozin maximum concentration and area under the concentration-time curve (AUC) values increased in a dose-dependent manner with the time to maximum concentration $\left(t_{\max }\right)$ of $1.0 \mathrm{~h}$ and elimination half-life $\left(t_{1 / 2}\right)$ of $10.22-13.26 \mathrm{~h}$ on Day 1. No significant changes in $t_{\max }$ and $t_{1 / 2}$ were observed after multiple-dose administration. The linearity factors, as calculated from the ratios of $\mathrm{AUC}_{0-24 \mathrm{~h}}$ on Day 16 to $\mathrm{AUC}_{0-\infty}$ on Day 1, were close to 1 in all canagliflozin groups. Canagliflozin increased $\mathrm{UGE}_{0-24 \mathrm{~h}}$ (80-110 g/day with canagliflozin $\geq 100 \mathrm{mg}$ ) and decreased $\mathrm{RT}_{\mathrm{G}}$ from the first day of treatment; these effects were sustained during the entire period of multiple administration. No 
significant AEs were noted. Urine volume was slightly increased on Day 1, but subsequent changes after repeated doses for 14 days were small. Urinary sodium tended to be higher in the early treatment period, whereas no particular change was observed in serum osmolality and hematocrit.

Conclusion: Canagliflozin increased UGE, decreased $\mathrm{RT}_{\mathrm{G}}$, and was well tolerated throughout the entire period of multiple administrations in Japanese patients with T2DM.

Funding: Mitsubishi Tanabe Pharma Corporation.

Trial registration:

ClinicalTrials.gov\#NCT00707954.

Keywords: Canagliflozin; Dehydration; Japanese patients; Pharmacodynamics; Pharmacokinetics; Sodium glucose co-transporter 2 inhibitor; Type 2 diabetes mellitus; Urine volume

\section{INTRODUCTION}

Type 2 diabetes mellitus (T2DM) is caused by insufficient insulin action due to increased insulin resistance and/or impaired insulin secretion. In addition to these mechanisms, the role of kidney function in glycemic control has been noted. In the kidney, approximately $90 \%$ of filtered glucose is reabsorbed by the sodium glucose co-transporter (SGLT) 2 in the S1 segment of proximal tubules, and the residual glucose is reabsorbed by SGLT1 in the S2 and S3 segments [1]. These transporters reabsorb filtered glucose almost completely until the filtered load exceeds the transport capacity; the plasma glucose (PG) concentration at which this occurs is designated the renal threshold for glucose $\left(\mathrm{RT}_{\mathrm{G}}\right)$. In patients with
T2DM, $\mathrm{RT}_{\mathrm{G}}$ is higher than that in nondiabetic individuals $[1,2]$.

The pharmacological inhibition of SGLT2 lowers $\mathrm{RT}_{\mathrm{G}}$ through the suppression of glucose reabsorption in the proximal tubules and exerts antihyperglycemic effects by inducing urinary glucose excretion (UGE). Thus, SGLT2 inhibitors reduce PG levels in an insulin-independent manner and also promote weight reduction by excreting the ingested calories in the form of glucose [3, 4]. Furthermore, some SGLT2 inhibitors have shown antihypertensive effects because of their diuretic action [5, 6]. In addition to blood glucose control, the management of body weight and blood pressure is critical for the prevention of diabetic complications [7, 8]. Therefore, SGLT2 inhibitors may offer a novel option for the treatment of T2DM. On the other hand, increased hematocrit level and transient dehydration after the administration of SGLT2 inhibitors have been reported $[6,9,10]$, whereas the changes in parameters related to body fluid volume after drug administration have not been investigated in detail in Japanese patients with T2DM.

Canagliflozin is an SGLT2 inhibitor available for the treatment of T2DM in many countries, including Japan [11-18]. In the United States, a clinical study was conducted on the tolerability and dose-dependent pharmacokinetics and pharmacodynamics of canagliflozin at doses of $50-300 \mathrm{mg}$ in healthy subjects or patients with T2DM [19, 20]. We previously reported the pharmacodynamic profiles of canagliflozin in Japanese patients with T2DM and moderate renal impairment in an open-label, cross-over study [14]. In the present study, we evaluated the pharmacokinetics, pharmacodynamics, and safety-including parameters related to body fluid volume-of canagliflozin in Japanese patients with T2DM. 


\section{METHODS}

\section{Patients}

Eligible patients included men and postmenopausal or surgically sterilized women aged 25-65 years diagnosed with T2DM at least 3 months before screening. Inclusion criteria were as follows: glycosylated hemoglobin [HbA1c (National Glycohemoglobin Standardization Program, NGSP)] 6.9-10.5\%; body mass index (BMI) of $18.5-39.9 \mathrm{~kg} / \mathrm{m}^{2}$ at the screening visit; fasting PG (FPG) of $140-270 \mathrm{mg} / \mathrm{dL}$; systolic blood pressure of 95-160 mmHg; diastolic blood pressure of $50-100 \mathrm{mmHg}$ with a pulse rate of $\geq 50 \mathrm{bpm}$; and no antidiabetic medication within 2 weeks prior to administration of canagliflozin. Patients were required to have undergone diet and exercise therapy, with no change in their regimen, for $\geq 3$ months before the study. Exclusion criteria were as follows: history of type 1 diabetes mellitus (DM) or secondary DM; the use of thiazolidinediones, insulin, thiazide diuretics, $\beta$-blockers, systemic steroids, nonsteroidal anti-inflammatory drugs, or anticoagulants within 3 months before consent; a history of, or current, serious diabetic complications requiring treatment; repeated severe hypoglycemic episodes; concurrent disorders that may cause gastrointestinal bleeding; skin symptoms requiring pharmacotherapy or a history of photosensitivity; serum creatinine level above the upper limit specified in the study center; a history of, or concurrent, diseases affecting renal function; eating disorders; a history of drug and/or food allergies; a history of surgeries that could affect gastrointestinal drug absorption; the consumption of grapefruit or cranberry (fruits or juices) within 1 week or food containing St John's Wort within 2 weeks before the first dose of the study drug; medical history or sign of heart disease; alcohol or drug abuse and/or smoking; positive for hepatitis B antigen, syphilis, hepatitis C antibody, or human immunodeficiency virus antibody; collection or donation of $\geq 400 \mathrm{~mL}$ of blood within 12 weeks or $\geq 200 \mathrm{~mL}$ of blood within 4 weeks before study entry; or participated in another clinical trial and received an investigational drug.

The study was conducted in accordance with ethical principles that have their origin in the Declaration of Helsinki, the Pharmaceutical Affairs Law of Japan, Good Clinical Practice, and the approved study protocol. Approval was obtained from institutional review boards and independent ethics committees for all participating centers. All patients provided written informed consent prior to participation.

\section{Study Design, Treatments and Blinding}

The study design is shown in Fig. 1a. This randomized, double-blind, placebo-controlled, multiple-dose, phase 1 study was conducted at four study centers in Japan. This study consisted of a washout period of 12 or more days to allow patients to discontinue from their previous antihyperglycemic agents, a single-day treatment of canagliflozin followed by a washout day, and 14 consecutive days of treatment. Patients who met inclusion criteria were randomized using a block allocation method into one of five groups to receive canagliflozin (1 of 4 doses) or placebo. Following an overnight fast, patients were given a placebo in a single-blind fashion QD and underwent baseline safety and pharmacodynamic assessments on Day 0 (pretreatment baseline) and received double-blind treatment with canagliflozin (25, 
(a)

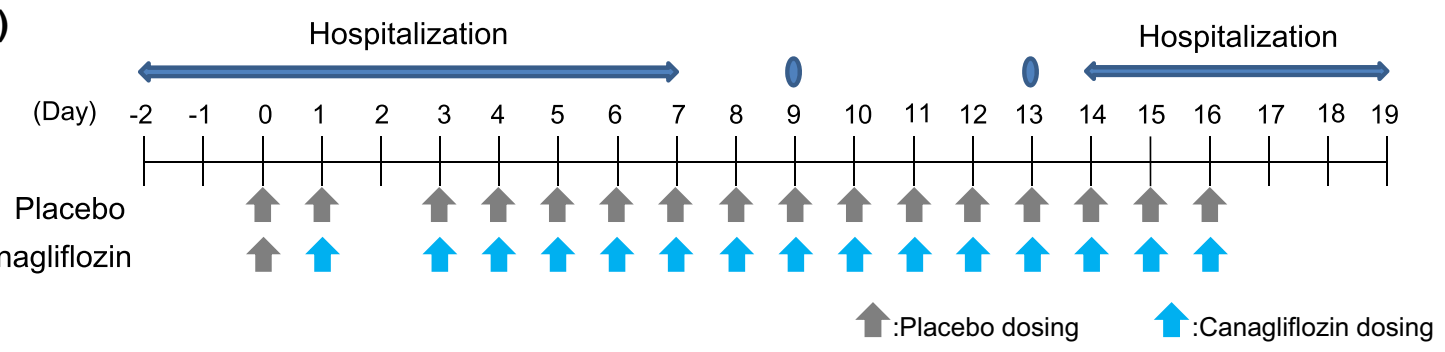

(b)

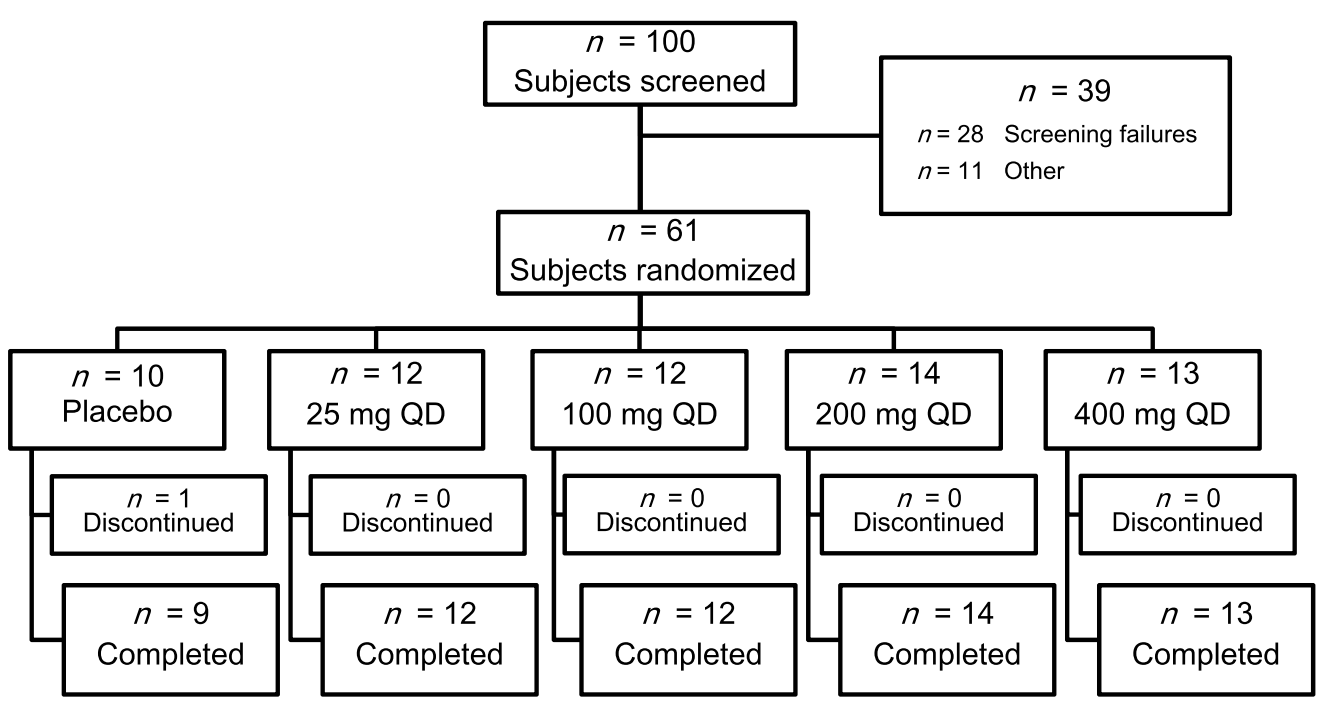

Fig. 1 Study design (a) and participant flow (b)

100 , 200, or $400 \mathrm{mg}$ QD) or placebo on Day 1 and Days 3-16. The first dose was administered between 8:00 and 9:00 a.m. Within $10 \mathrm{~min}$ of dosing, patients received a standardized breakfast; thereafter, standardized lunch and dinner were provided 4.5 and $10.5 \mathrm{~h}$ post-dosing, respectively. Patients were admitted to the clinical research unit on Day 2 , discharged on Day 7, and visited the unit on Days 9 and 13. Patients were admitted again on Day 14, discharged on Day 19, and returned for safety assessments later.

\section{Sampling and Analysis}

Pharmacokinetic parameters were assessed using venous blood samples at different time points: 0 (before drug administration), 0.5, 1, $1.5,2,3,4,6,8$, and $12 \mathrm{~h}$ after administration on Days 1 and 16; 0 h on Days 3, 5, 7, 9, 13, and 15; and before breakfast on Days 2, 17, 18, and 19. Pharmacodynamic parameters were assessed using venous blood samples at the following time points: $0,0.5,1.5,2.5,4.5,6,8,10.5,11$, 12,13 , and $14 \mathrm{~h}$ after administration on Days 1 and 16; $0 \mathrm{~h}$ on Days 3, 5, 7, 9, 13, and 15; and before breakfast on Days 2,17 and 18. Urine samples for the assessment of UGE were collected at intervals as specified: 0-2, 2-4.5, 4.5-7, 7-10.5, 10.5-13, and 13-24 h on Days 0, 1, and 16. On Days 2, 3, 4, 5, 6, 15, 17, and 18, urine was collected over a period of $0-24 \mathrm{~h}$.

\section{Pharmacokinetic Assessments}

After solid-phase extraction with Oasis ${ }^{\circledR}$ HLB (Waters Corporation, Milford, MA, USA), plasma and urinary canagliflozin 
concentrations were determined using high-performance liquid chromatography/tandem mass spectrometry (API $4000^{\mathrm{TM}}$, SCIEX, Framingham, MA, USA), with ${ }^{13} \mathrm{C}_{6}$-canagliflozin as an internal standard. The validated quantification range was $1-2000 \mathrm{ng} / \mathrm{mL}$. All validation results satisfied the predefined acceptance criteria.

\section{Pharmacodynamic Assessments}

HbA1c, urinary glucose, PG, and serum insulin concentrations were assessed for pharmacodynamics evaluation. On Days 2 and 17, changes from baseline (Day 1) were determined for FPG and fasting serum insulin. On Days 1 and 16, changes from baseline (Day 0 ) were determined for 24-h mean PG (MPG) and 24-h mean serum insulin.

Changes from baseline in UGE were assessed on Days $0,1,2,3,4,5,6,15,16,17$, and 18 . The $\mathrm{RT}_{\mathrm{G}}$ on Days 0,1 , and 16 was calculated post hoc from the measured PG, UGE, and estimated glomerular filtration rate as previously described [18, 21, 22]. HbA1c values were measured with the Japan Diabetic Society (JDS) method during the study. As JDS issued a recommendation to use HbA1c (NGSP) instead of HbA1c (JDS) after study completion [23], HbA1c (JDS) values were converted to HbA1c (NGSP) in the present report.

\section{Safety Assessments}

Adverse events (AEs) were classified according to the system organ class and preferred terms of the Medical Dictionary for Regulatory Activity (MeDRA/J version 11.1). In addition, volume-related safety was assessed with changes in blood and urinary laboratory parameters and vital signs, including blood pressure, pulse rate, and body temperature.

\section{Statistical Analysis}

For sample size determination, the number of subjects was set at 15 (12 receiving canagliflozin and 3 receiving placebo) for each step to evaluate pharmacokinetics, pharmacodynamics, and safety of canagliflozin administered as single and 14-day multiple doses. As reference, assuming that the percentage change in $\mathrm{MPG}_{0-24 \mathrm{~h}}$ on Day 16 relative to the day of placebo administration is comparable to that obtained in the clinical studies conducted in other countries, the probability of detecting a difference between the highest dose $(400 \mathrm{mg}$ ) treatment and placebo was $99 \%$, whereas the probability of detecting a difference between the 200-mg treatment and placebo was 96\% (two-sided test with a significance level of 5\%). Safety analyses were performed in all patients who received $\geq 1$ dose of the study drug. Pharmacokinetic and pharmacodynamic analyses were conducted in the subsets of subjects in the safety analysis set who were evaluable for the pharmacokinetic and pharmacodynamic assessments, respectively. For the pharmacokinetic analysis, WinNonlin ver.5.2, Pharsight, Mountain View, CA, USA) was used; for all other analysis, SAS ${ }^{\circledR}$ ver. 9.1.3 (SAS Institute Inc., Cary, NC, USA) was used.

\section{RESULTS}

\section{Patient Disposition and Baseline Characteristics}

A total of 61 patients (50 men and 11 women) were randomized to receive canagliflozin 
Table 1 Patient disposition and baseline characteristics

\begin{tabular}{|c|c|c|c|c|c|c|}
\hline & \multirow[t]{2}{*}{ Placebo $(n=10)$} & \multicolumn{4}{|l|}{ Canagliflozin } & \multirow[t]{2}{*}{ Total $(N=61)$} \\
\hline & & $\begin{array}{l}25 \mathrm{mg} \\
(n=12)\end{array}$ & $\begin{array}{l}100 \mathrm{mg} \\
(n=12)\end{array}$ & $\begin{array}{l}200 \mathrm{mg} \\
(n=14)\end{array}$ & $\begin{array}{l}400 \mathrm{mg} \\
(n=13)\end{array}$ & \\
\hline \multicolumn{7}{|l|}{ Gender, $n(\%)$} \\
\hline Male & $8(80)$ & $8(66.7)$ & $12(100)$ & $13(92.9)$ & $9(69.2)$ & $50(82.0)$ \\
\hline Female & $2(20)$ & $4(33.3)$ & $0(0)$ & $1(7.1)$ & $4(30.8)$ & $11(18.0)$ \\
\hline \multicolumn{7}{|l|}{ Mean (SD) } \\
\hline Age, years & $57.6(6.3)$ & $49.0(10.6)$ & $52.1(7.6)$ & $56.2(8.6)$ & $56.5(8.2)$ & $54.3(8.8)$ \\
\hline Weight, kg & $69.73(14.08)$ & 74.24 (11.04) & $73.44(11.07)$ & $63.67(13.62)$ & $73.88(10.18)$ & $70.84(12.38)$ \\
\hline BMI, $\mathrm{kg} / \mathrm{m}^{2}$ & $25.75(3.37)$ & $26.41(2.39)$ & $25.25(2.40)$ & $23.20(4.03)$ & $27.44(3.13)$ & $25.56(3.39)$ \\
\hline $\mathrm{FPG}, \mathrm{mg} / \mathrm{dL}$ & $184.9(35.8)$ & $172.2(19.2)$ & $162.5(20.4)$ & $163.4(14.3)$ & $170.9(27.2)$ & $170.1(24.3)$ \\
\hline HbAlc, \% & $8.91(1.16)$ & $9.11(0.85)$ & $8.28(0.85)$ & $8.51(0.82)$ & $8.58(0.75)$ & $8.66(0.90)$ \\
\hline
\end{tabular}

FPG: $1 \mathrm{mg} / \mathrm{dL}=0.0555 \mathrm{mmol} / \mathrm{L}$

$B M I$ body mass index, $F P G$ fasting plasma glucose, $S D$ standard deviation

( $n=51)$ or placebo $(n=10)$ in the study (Fig. $1 b)$. The mean \pm standard deviation (SD) of age, body weight, BMI, FPG, and HbA1c of these 61 patients were $\quad 54.3 \pm 8.8$ years, $\quad 70.84 \pm 12.38 \mathrm{~kg}$, $25.56 \pm 3.39 \mathrm{~kg} / \mathrm{m}^{2}, \quad 170.1 \pm 24.3 \mathrm{mg} / \mathrm{dL}, \quad$ and $8.66 \% \pm 0.90 \%$, respectively (Table 1 ). No remarkable differences were observed in these parameters across subgroups. Twelve patients had received pharmacotherapy for T2DM prior to participation. Sixty subjects completed the study, except one in the placebo group. Fifty-one patients (42 men and 9 women) were assigned to the canagliflozin groups and were subjected to pharmacokinetic analysis. The mean \pm SD of age, body weight, and BMI of these 51 patients were $53.6 \pm 9.1$ years, $71.06 \pm 12.17 \mathrm{~kg}$, and $25.52 \pm 3.43 \mathrm{~kg} / \mathrm{m}^{2}$, respectively.

\section{Pharmacokinetics}

Canagliflozin was administered to patients at $25,100,200$, or $400 \mathrm{mg}$ in a single dose (Day 1), followed by a 1-day washout (Day 2) and repeated doses for 14 days (Days 3-16, Fig. 1a). Figure 2 shows the plasma canagliflozin concentration-time profile on Days 1 and 16 (the day of last administration). The plasma concentrations of canagliflozin rapidly increased after oral administration and declined in a biphasic manner. On Days 1 and $16, t_{\max }$ was 1.0 and $1.0-1.3 \mathrm{~h}$, respectively, and elimination half-life $\left(t_{1 / 2}\right)$ was $10.22-13.26 \mathrm{~h}$ and $11.81-16.16 \mathrm{~h}$, respectively (Table 2 ). No marked differences were observed in $t_{\max }$ and $t_{1 / 2}$ values between Days 1 and 16 . The $C_{\max }$ and AUC $_{0-24 \mathrm{~h}}$ increased in a dose-dependent manner on both Days 1 and 16. The mean accumulation ratios (ARs) obtained by dividing the $\mathrm{AUC}_{0-24 \mathrm{~h}}$ on Day 16 by the $\mathrm{AUC}_{0-24 \mathrm{~h}}$ on Day 1 were in the range 1.19-1.42. A trend toward increased ratios was observed with dose increase. The linearity factors obtained by dividing the $\mathrm{AUC}_{0-24 \mathrm{~h}}$ on Day 16 by the $\mathrm{AUC}_{0-\infty}$ on Day 1 were approximately 1 at any dose of canagliflozin $(25,100,200$, and $400 \mathrm{mg}$ ). The mean ratios of trough 


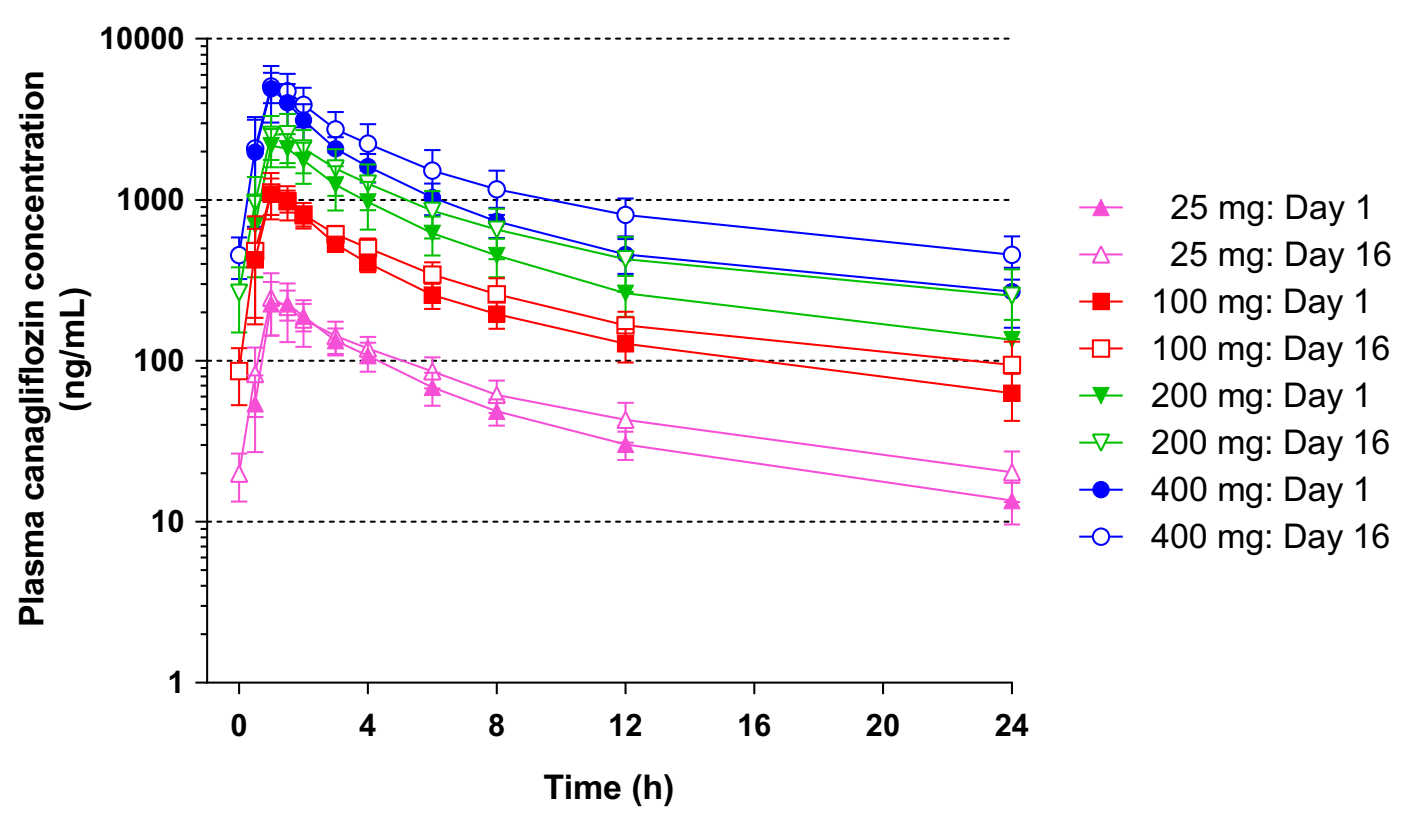

Fig. 2 Plasma concentration-time profiles after canagliflozin treatment on Days 1 and 16. Data are presented as the mean \pm standard deviation

concentrations at each dose to the concentration just before drug administration on Day 16 were in the range $0.93-1.00$ on Day 7 (4 days after the initiation of repeated administration). Thereafter, the ratios became nearly constant irrespective of the dose, suggesting that the plasma concentration of canagliflozin reaches steady state by Day 7 . The means of the 24-h urinary excretion rate of unchanged canagliflozin at each dose on Days 1 and 16 were approximately $0.4 \%$ and $0.5-0.6 \%$, respectively (Table 2 ).

\section{Pharmacodynamic Effects}

The mean $\mathrm{UGE}_{0-24 \mathrm{~h}}$ values were approximately 14-24 g on Day 0 and markedly increased after the administration of canagliflozin at 25, 100, 200 , or $400 \mathrm{mg}$ compared with baseline, whereas no increase was observed in $\mathrm{UGE}_{0-24 \mathrm{~h}}$ after placebo administration. The mean change from baseline in $\mathrm{UGE}_{0-24 \mathrm{~h}}$ values on Day 1 was approximately $60 \mathrm{~g} /$ day in the $25-\mathrm{mg}$ group and ranged from 80 to $110 \mathrm{~g} /$ day with no great difference in the 100-400-mg groups (Fig. 3a). Similar increases from baseline in $\mathrm{UGE}_{0-24 \mathrm{~h}}$ were observed on Day 16, indicating that the increase in UGE because of canagliflozin is sustained during repeated-dose administration. The increase in UGE was detected during 24-48 and $48-72 \mathrm{~h}$ after the last administration of canagliflozin at $\geq 100 \mathrm{mg}$. There was no increase in $\mathrm{UGE}_{0-24 \mathrm{~h}}$ during placebo administration.

The mean baseline $\mathrm{RT}_{\mathrm{G} 0-24 \mathrm{~h}}$ values on Day 0 ranged from 210 to $250 \mathrm{mg} / \mathrm{dL}$ in the canagliflozin and placebo groups, which were higher than those in healthy adults $(\sim 200 \mathrm{mg}$ / dL) [1]. The $\mathrm{RT}_{\mathrm{GO}-24 \mathrm{~h}}$ decreased after the administration of canagliflozin on both Days 1 and 16 (Fig. 3b). The $\mathrm{RT}_{\mathrm{G}}$-lowering effects of canagliflozin did not diminish after repeated-dose administration. No marked difference was observed in groups that received canagliflozin $\geq 100 \mathrm{mg}$. Changes from baseline in $\mathrm{MPG}_{0-24 \mathrm{~h}}$ on Days 1 and 16, and those in FPG on Days 2 and 17 were greater in 
Table 2 Pharmacokinetic parameters

\begin{tabular}{|c|c|c|c|c|}
\hline \multirow{2}{*}{ Parameters, mean (SD) } & \multicolumn{4}{|l|}{ Canagliflozin } \\
\hline & $25 \mathrm{mg}(n=12)$ & $100 \mathrm{mg}(n=12)$ & $200 \mathrm{mg}(n=14)$ & $400 \mathrm{mg}(n=13)$ \\
\hline \multicolumn{5}{|l|}{ Day 1} \\
\hline$C_{\max }(\mathrm{ng} / \mathrm{mL})$ & $248(55)$ & $1126(228)$ & $2304(539)$ & $5028(1733)$ \\
\hline $\mathrm{AUC}_{0-24 \mathrm{~h}}(\mathrm{ng} \cdot \mathrm{h} / \mathrm{mL})$ & $1295(240)$ & $5468(899)$ & $11,991(3042)$ & $21,836(4983)$ \\
\hline$t_{\max }(\mathrm{h})^{\mathrm{a}}$ & $1.0[1.0-2.0]$ & $1.0[1.0-1.5]$ & $1.0[1.0-1.5]$ & $1.0[1.0-3.0]$ \\
\hline$t_{1 / 2}(\mathrm{~h})$ & $10.70(1.76)$ & $10.22(1.89)$ & $13.26(4.96)$ & $12.15(2.34)$ \\
\hline Urinary excretion rate $(\%)^{\mathrm{b}}$ & $0.387(0.070)$ & $0.384(0.084)$ & $0.424(0.107)$ & $0.385(0.097)$ \\
\hline \multicolumn{5}{|l|}{ Day 16} \\
\hline$C_{\max }(\mathrm{ng} / \mathrm{mL})$ & $263(91)$ & $1136(330)$ & $2688(805)$ & $5236(1123)$ \\
\hline $\mathrm{AUC}_{0-24 \mathrm{~h}}(\mathrm{ng} \cdot \mathrm{h} / \mathrm{mL})$ & $1556(387)$ & $6635(1367)$ & $16,716(5464)$ & $30,766(7916)$ \\
\hline$t_{\max }(\mathrm{h})^{\mathrm{a}}$ & $1.0[1.0-3.0]$ & $1.0[1.0-1.5]$ & $1.3[1.0-1.5]$ & $1.0[1.0-1.5]$ \\
\hline$t_{1 / 2}(\mathrm{~h})$ & $14.21(7.46)$ & $11.81(3.22)$ & $16.16(5.87)^{\mathrm{c}}$ & $15.76(5.56)^{d}$ \\
\hline Urinary excretion rate $(\%)^{\mathrm{b}}$ & $0.599(0.183)$ & $0.641(0.204)$ & $0.614(0.195)$ & $0.495(0.153)$ \\
\hline $\mathrm{AR}$ & $1.19(0.12)$ & $1.21(0.12)$ & $1.38(0.17)$ & $1.42(0.29)$ \\
\hline
\end{tabular}

$A R$ accumulation ratio, $A U C_{O-24 h}$ area under the concentration-time curve from time zero to $24 \mathrm{~h}, C_{\max }$ maximum concentration, $S D$ standard deviation, $t_{1 / 2}$ elimination half-life, $t_{\max }$ time to maximum concentration

a Median [Min-Max]

b $0-24 \mathrm{~h}$

c $n=13$

d $n=12$
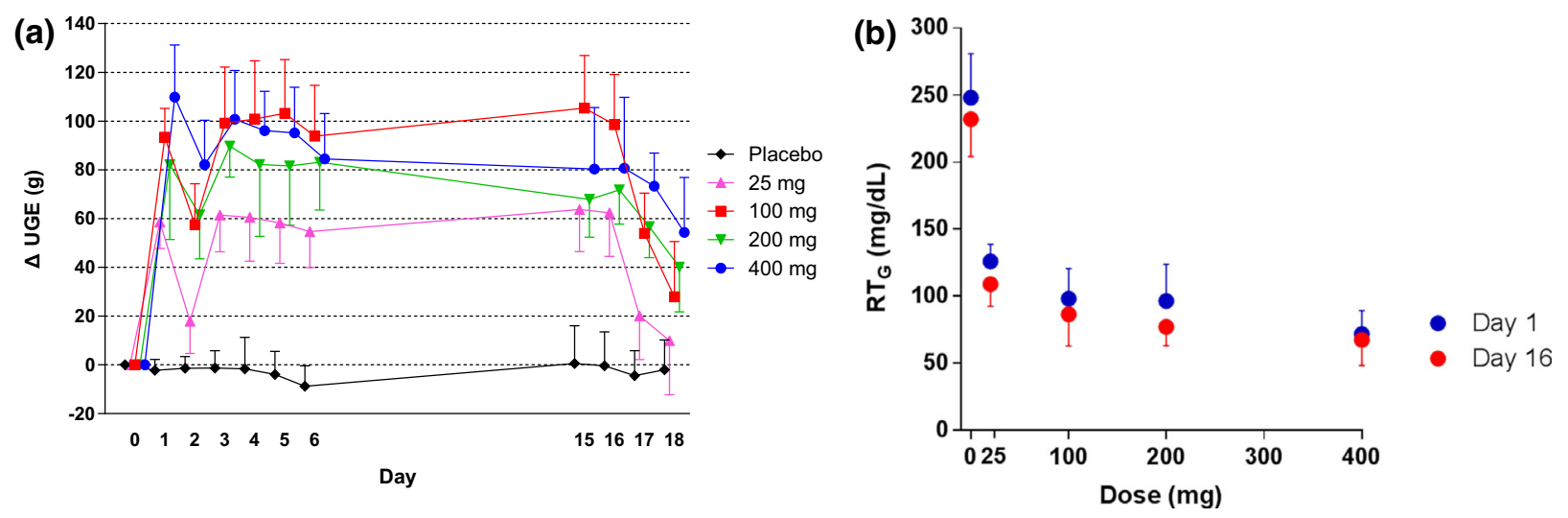

Fig. 3 Effect of canagliflozin on changes in 24-h UGE (a) and 24-h mean $\mathrm{RT}_{\mathrm{G}}$ (b). Data are mean \pm standard deviation. $U G E$ urinary glucose excretion, $R T_{G}$ renal threshold for glucose

canagliflozin-treated groups compared with the placebo group. Fasting serum insulin tended to decrease in groups that received canagliflozin $\geq 100 \mathrm{mg}$. The 24-h mean concentration of insulin also tended to decrease in canagliflozin-treated groups (see 
Table S1 in the Electronic Supplementary Material).

\section{Safety}

Of the AEs observed in a double-blind manner, those reported in $\geq 2$ cases were as follows: occult blood positive [canagliflozin groups: 14 cases in $12(23.5 \%)$ of 51 patients; placebo group: 4 cases in 3 (30.0\%) of 10 patients], diarrhea [canagliflozin groups: 6 cases in 5 (9.8\%) of 51 patients; placebo group: 4 cases in $2(20.0 \%)$ of 10 patients], anemia [canagliflozin groups: 4 cases in $4(7.8 \%)$ of 51 patients; placebo group: 1 case in $1(10.0 \%)$ of 10 patients], urine ketone body present [canagliflozin groups: 3 cases in 3 (5.9\%) of 51 patients; placebo group: 0 case $(0.0 \%)$ of 10 patients], dizziness [canagliflozin groups: 2 cases in $2(3.9 \%)$ of 51 patients; placebo group: 0 case $(0.0 \%)$ of 10 patients], toothache [canagliflozin groups: 2 cases in 2 (3.9\%) of 51 patients; placebo group: 1 case in $1(10.0 \%)$ of 10 patients], and nasopharyngitis [canagliflozin groups: 1 case in 1 (2.0\%) of 51 patients; placebo group: 3 cases in 3 (30.0\%) of 10 patients]. AEs related to skin disorders were not observed in this study.

At baseline, the mean 24-h urine volume was approximately $2.6-3.3 \mathrm{~L}$ in all groups (see Fig. S1 in the Electronic Supplementary Material). Changes in urine volume and water intake from the baseline are shown in Fig. 4a, b, respectively. In canagliflozin groups, the 24-h urine volume slightly increased on Day 1, but subsequent changes during the 14-day repeated-dose administration period were small. Water intake increased or decreased but
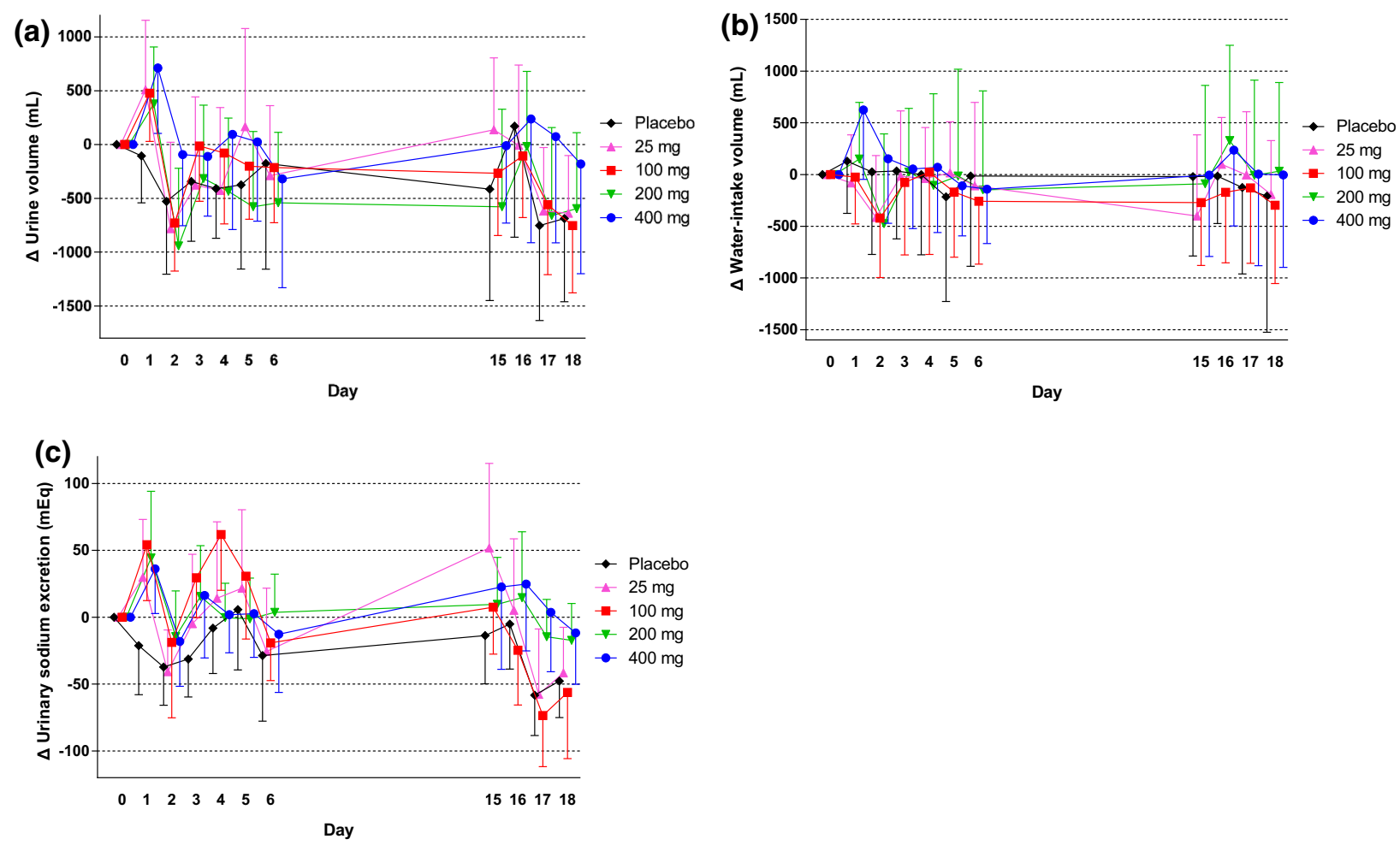

Fig. 4 Effects of canagliflozin on the changes from baseline in urinary volume (a), water intake (b), and urinary sodium excretion (c). Data are mean \pm standard deviation 


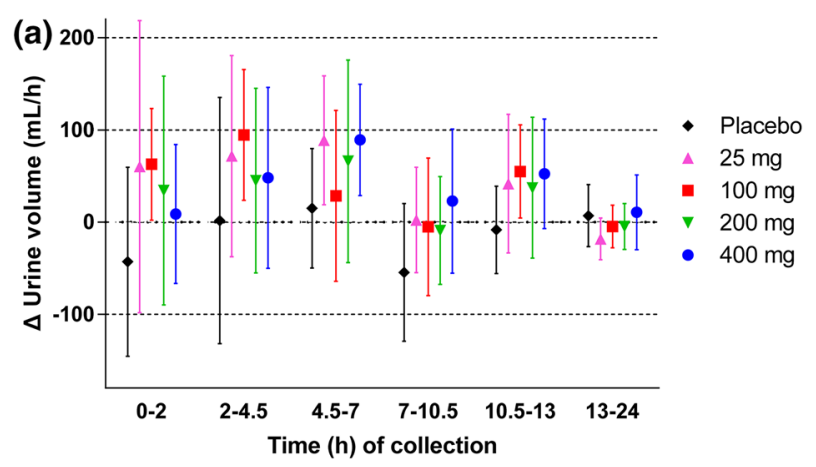

(b)

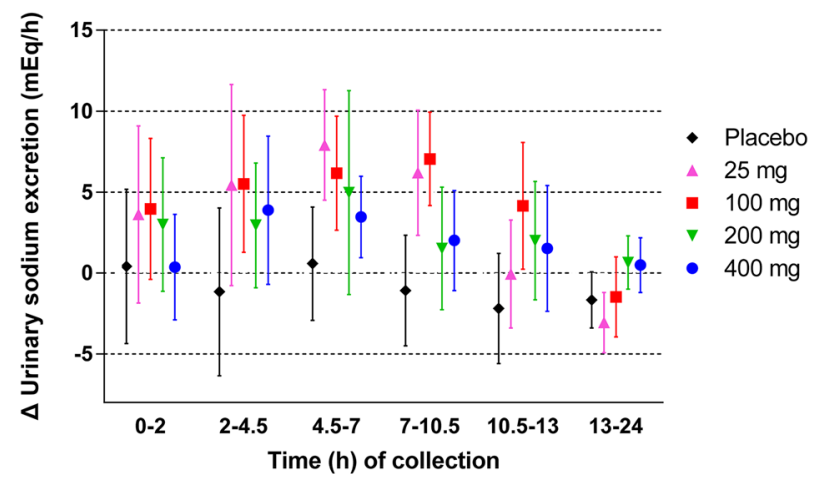

(c)

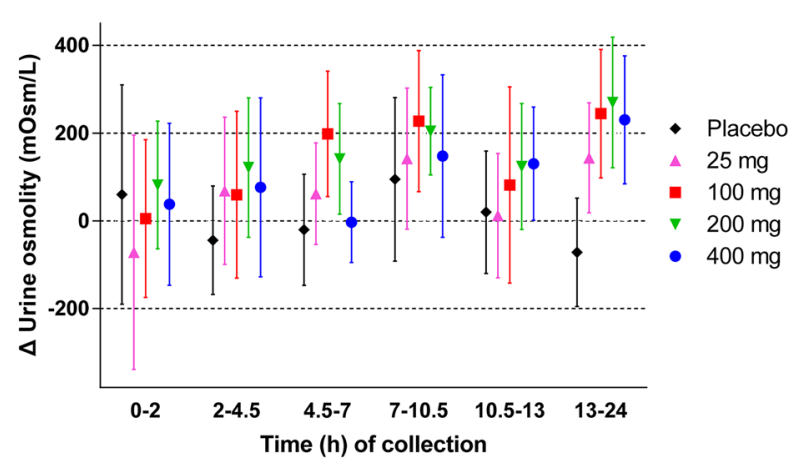

Fig. 5 Change from baseline on Day 1 in urine volume (a), sodium excretion (b), and urine osmolality (c). Data are mean \pm standard deviation

did not markedly change during this period (Fig. 4b). No remarkable changes were observed in the urinary excretion of electrolytes, including potassium, chloride, calcium, magnesium, and inorganic phosphorus, but not sodium, in canagliflozin-treated groups compared with the placebo group (see Table S2 in the Electronic Supplementary Material). A transient nominal increase of sodium was observed on Day 1, although this increase reversed within a week (Fig. 4c). The change in hourly urine volume over time from baseline on Day 1 is shown in Fig. $5 \mathrm{a}$. The hourly urine volume was slightly increased in canagliflozin-treated groups compared with the placebo group up to $10.5-13 \mathrm{~h}$, whereas no difference was seen at the 13-24-h time period. The changes in hourly urinary sodium excretion from baseline on Day 1 showed similar time profiles as those for hourly urine volume (Fig. 5b). The change in urine osmolality over time from baseline on Day 1 showed a trend toward an increase in the canagliflozin-treated groups, whereas no change in osmolality was observed in the placebo group (Fig. 5c). The increase in urine osmolality was sustained up to Day 16 in canagliflozin-treated groups consistent with the persistent increase in UGE (data not shown). On the other hand, serum osmolality was maintained constant during the treatment period in all study groups (Fig. 6a). The hematocrit level was similar between the canagliflozin and placebo groups at baseline and remained unchanged during the study period (Fig. 6b). Mean systolic and diastolic blood pressures tended to decrease in groups that received canagliflozin $\geq 100 \mathrm{mg}$ (see Fig. S2 

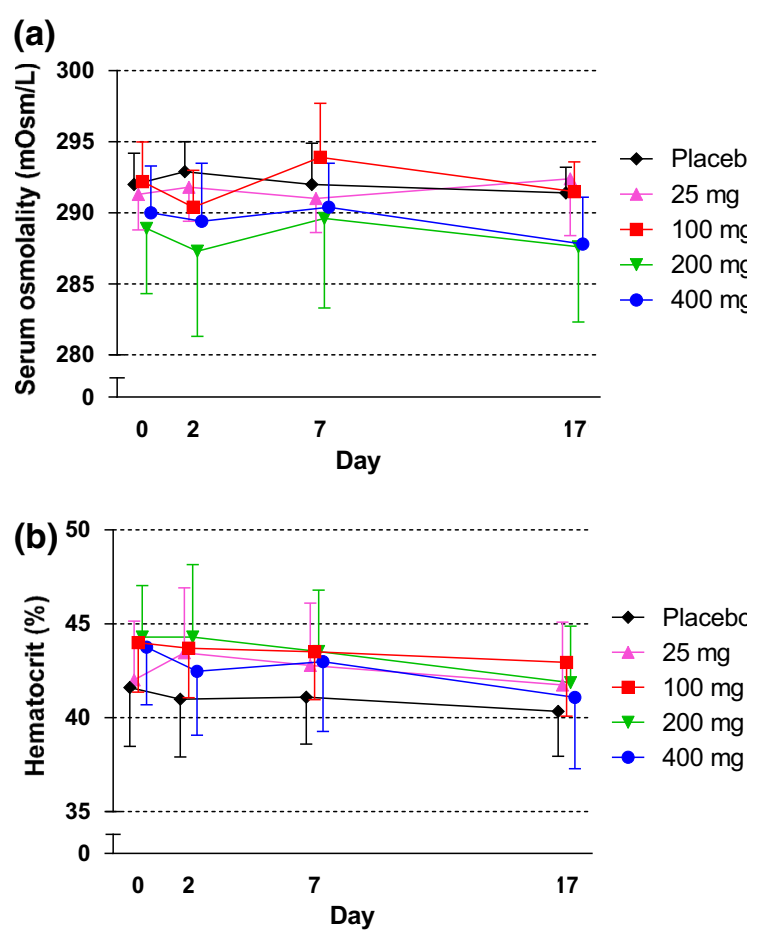

Fig. 6 Effects of canagliflozin on serum osmolality (a) and hematocrit (b). Data are mean \pm standard deviation

in the Electronic Supplementary Material). No increases were observed in the pulse rate and the incidence of orthostatic hypotension (data not shown).

\section{DISCUSSION}

The $C_{\max }$ and AUC values of plasma canagliflozin concentrations increased in a dose-dependent manner on Days 1 and 16. No marked changes were observed in $t_{\max }$ and $t_{1 / 2}$ after multiple doses of canagliflozin. The mean ARs and linearity factors were close to 1 in all canagliflozin groups, and the trough plasma concentration of canagliflozin reached steady state by Day 7 for all doses. These results suggest that there is no accumulation of canagliflozin in plasma. No significant changes in renal clearance were observed after multiple doses at all dose levels. These pharmacokinetic profiles of canagliflozin in Japanese patients were similar to those previously reported in non-Japanese patients $[19,20]$.

Renal glucose reabsorption was rapidly inhibited, and $\mathrm{RT}_{\mathrm{G}}$ was decreased after canagliflozin administration on Days 1 and 16 . A near-maximal $\mathrm{RT}_{\mathrm{G}}$-lowering effect was induced in the 100-mg group. The $\mathrm{RT}_{\mathrm{G}}$-lowering effects of canagliflozin were not diminished by repeated administration. UGE was markedly increased in all canagliflozin-treated groups. The change in $\mathrm{UGE}_{0-24 \mathrm{~h}}$ from baseline was smallest in the 25 -mg group, and ranged from 80 to $110 \mathrm{~g} /$ day with no great difference in the $100-400-\mathrm{mg}$ groups. Collectively, it is noted that canagliflozin exerts near-maximal efficacy at a dose of $100 \mathrm{mg}$ in Japanese patients with T2DM. The increase in UGE was sustained during repeated-dose administration. The decrease in both $\mathrm{MPG}_{0-24 \mathrm{~h}}$ and FPG after single and repeated doses was greater in the canagliflozin groups than in the placebo group. These pharmacodynamic profiles of canagliflozin in Japanese patients were also similar to those in studies with non-Japanese patients $[3,20,22$, 24].

In the current short-term study, canagliflozin was well tolerated in patients with T2DM, with a low incidence of AEs. Urinary ketones were observed in 3 patients in canagliflozin-treated groups; 2 cases at a dose of $200 \mathrm{mg}$ and 1 case at a dose of $400 \mathrm{mg}$. All cases were mild and rapidly returned to a negative level without signs and symptoms of ketoacidosis. It is recommended that patients taking SGLT2 inhibitors should be assessed for ketoacidosis when they present with signs and symptoms of metabolic acidosis to prevent delayed diagnosis and management $[25,26]$. We then assessed any influences on urine volume, urine electrolytes, hematocrit, and 
serum osmolality in patients with T2DM. In canagliflozin-treated groups, although an increasing trend was observed in the 24-h urine volume on Day 1, this increase soon declined toward baseline levels despite the sustained UGE increase, which is similar to previously reported findings [22]. On Day 1, hourly urine volume was slightly increased in the canagliflozin-treated groups compared with the placebo group up to $10.5-13 \mathrm{~h}$, whereas no increase was observed thereafter. Interestingly, this time-course profile of urine volume on Day 1 was similar to that of urinary sodium excretion, suggesting the development of natriuresis during the early stages of canagliflozin treatment. There is a discrepancy between the time-course of urinary sodium increase and urinary glucose excretion. The diuretic effects of a natriuretic antihypertensive agent, such as chlorothiazide, persist only for about 1 week because of a compensatory mechanism against diuresis [27]. Therefore, a similar compensatory mechanism against canagliflozin-induced diuresis may be induced in the later stages. Further investigations remain to be addressed. Volume-related parameters, including mean hematocrit values and serum osmolality, were not markedly changed in the present study although some SGLT2 inhibitors have been reported to increase hematocrit values and transiently decrease body fluid volumes $[5,9$, 10].

A limitation of this study was that the treatment period was relatively short compared with previous studies of canagliflozin and the comparatively small sample size of female subjects (11 females vs 50 males). Studies with longer treatment periods and more female cases are required to address the effect of canagliflozin on body fluid volume.

\section{CONCLUSIONS}

In Japanese patients with T2DM in the present study, the pharmacokinetic profiles of canagliflozin were similar to those in non-Japanese patients. The maximal pharmacodynamic effect was exerted at canagliflozin doses $\geq 100 \mathrm{mg}$, and it was well tolerated in this population.

\section{ACKNOWLEDGMENTS}

The authors thank H. Kubo, MD, PhD, for insightful discussions. The authors thank all investigators, study teams, and patients for participating in this study. Canagliflozin was developed by Mitsubishi Tanabe Pharma Corp. in collaboration with Janssen Research \& Development, LLC. This study was funded by Mitsubishi Tanabe Pharma Corp. The authors thank Dr. A. Saito, of Tanabe R\&D Service Co., Ltd., for providing editorial support, which was funded by Mitsubishi Tanabe Pharma Corp. Article processing charges, and the open access charge for this study were funded by Mitsubishi Tanabe Pharma Corp. All authors had full access to all of the data in this study and take complete responsibility for the integrity of the data and accuracy of the data analysis.

Study investigators: Dr. Satoshi Inoue, Medical Corporation Heishinkai OCROM Clinic; Medical Corporation Heishinkai ToCROM Clinic; Dr. Kenichi Furihata, P-One Clinic, Keikokai Medical Corporation; Dr. Takeshi Osonoi, Internal Medicine Department, Nakakinen Clinic.

H. Iijima contributed to the preparation of the manuscript, interpretation and discussion of the data. N. Maruyama contributed to the development of the protocol and study design, and data collection. T. Kifuji contributed to the 
interpretation and discussion of the pharmacokinetic/pharmacodynamic data, and $\mathrm{N}$. Inagaki contributed to the interpretation and discussion of the results. All named authors meet the International Committee of Medical Journal Editors (ICMJE) criteria for authorship for this manuscript, take responsibility for the integrity of the work as a whole, and have given final approval to the version to be published.

Conflict of interest. N. Inagaki has received consulting fees and research support from Mitsubishi Tanabe Pharma Corp., and has served on speakers bureaus for Mitsubishi Tanabe Pharma Corp., ARKRAY, Inc., Astellas Pharma Inc., AstraZeneca K.K., Bayer Yakuhin, Ltd., Daiichi Sankyo Co., Ltd., Eisai Co., Ltd., Eli Lilly Japan K.K., Japan Tobacco Inc., Johnson \& Johnson K.K., Kowa Pharmaceutical Co., Ltd., Kissei Pharmaceutical Co., Ltd., Kyowa Hakko Kirin Co., Ltd., Medtronic, Inc., MSD K.K., Nippon Boehringer Ingelheim Co., Ltd., Novartis Pharma K.K., Novo Nordisk Pharma Ltd., Ono Pharmaceutical Co., Ltd., Roche Diagnostics K.K., Sanofi K.K., Sanwa Kagaku Kenkyusho Co., Ltd., Sumitomo Dainippon Pharma Co., Ltd., Taisho Toyama Pharmaceutical Co., Ltd., Takeda Pharmaceutical Co., Ltd., and Tsumura \& Co. He has also received consulting fees and/or research support from Astellas Pharma Inc., AstraZeneca K.K., Bristol-Myers K.K., Chugai Pharmaceutical Co., Ltd., Daiichi Sankyo Co., Ltd., Eisai Co., Ltd., Eli Lilly Japan K.K., GlaxoSmithKline K.K., Japan Tobacco Inc., Kissei Pharmaceutical Co., Ltd., Kyowa Hakko Kirin Co., Ltd., MSD K.K., Nippon Boehringer Ingelheim Co., Ltd., Nipro Corporation, Novartis Pharma K.K., Novo Nordisk Pharma Ltd., Ono Pharmaceutical Co., Ltd., Pfizer Japan Inc., Roche Diagnostics K.K., Sanofi K.K., Sanwa Kagaku Kenkyusho Co., Ltd., Shionogi \& Co.,
Ltd., Shiratori Pharmaceutical Co., Ltd., Sumitomo Dainippon Pharma Co., Ltd., Taisho Pharmaceutical Co., Ltd., Takeda Pharmaceutical Co., Ltd., Terumo Corporation., and Tsumura \& Co. H. Iijima and T. Kifuji are both employees of Mitsubishi Tanabe Pharma Corporation.

Compliance with ethics guidelines. All procedures followed were in accordance with the ethical standards of the relevant committee on human experimentation (institutional and national) and with the Helsinki Declaration of 1964, as revised in 2013. Informed consent was obtained from all patients for being included in the study.

Open Access. This article is distributed under the terms of the Creative Commons Attribution-NonCommercial 4.0 International License (http://creativecommons.org/licenses/bync/4.0/), which permits any noncommercial use, distribution, and reproduction in any medium, provided you give appropriate credit to the original author(s) and the source, provide a link to the Creative Commons license, and indicate if changes were made.

\section{REFERENCES}

1. Gerich JE. Role of the kidney in normal glucose homeostasis and in the hyperglycaemia of diabetes mellitus: therapeutic implications. Diabet Med. 2010;27:136-42.

2. Rave K, Nosek L, Posner J, Heise T, Roggen K, van Hoogdalem EJ. Renal glucose excretion as a function of blood glucose concentration in subjects with type 2 diabetes-results of a hyperglycaemic glucose clamp study. Nephrol Dial Transplant. 2006;21:2166-71.

3. Scheen AJ. Pharmacodynamics, efficacy and safety of sodium-glucose co-transporter type 2 (SGLT2) inhibitors for the treatment of type 2 diabetes mellitus. Drugs. 2015;75:33-59. 
4. Fujita Y, Inagaki N. Renal sodium glucose cotransporter 2 inhibitors as a novel therapeutic approach to treatment of type 2 diabetes: clinical data and mechanism of action. J Diabetes Investig. 2014;5:265-75.

5. Maliha G, Townsend RR. SGLT2 inhibitors: their potential reduction in blood pressure. J Am Soc Hypertens. 2015;9:48-53.

6. Baker WL, Smyth LR, Riche DM, Bourret EM, Chamberlin KW, White WB. Effects of sodium-glucose co-transporter 2 inhibitors on blood pressure: a systematic review and meta-analysis. J Am Soc Hypertens. 2014;8(262-75):e9.

7. Pi-Sunyer X, Blackburn G, Brancati FL, et al. Reduction in weight and cardiovascular disease risk factors in individuals with type 2 diabetes: one-year results of the look AHEAD trial. Diabetes Care. 2007;30:1374-83.

8. Tight blood pressure control and risk of macrovascular and microvascular complications in type 2 diabetes: UKPDS 38. UK Prospective Diabetes Study Group. BMJ. 1998;317:703-13.

9. Sha S, Polidori D, Heise T, et al. Effect of the sodium glucose co-transporter 2 inhibitor canagliflozin on plasma volume in patients with type 2 diabetes mellitus. Diabetes Obes Metab. 2014;16:1087-95.

10. Lambers Heerspink HJ, de Zeeuw D, Wie L, Leslie B, List J. Dapagliflozin a glucose-regulating drug with diuretic properties in subjects with type 2 diabetes. Diabetes Obes Metab. 2013;15:853-62.

11. Inagaki N, Kondo K, Yoshinari $\mathrm{T}$, Maruyama N, Susuta Y, Kuki H. Efficacy and safety of canagliflozin in Japanese patients with type 2 diabetes: a randomized, double-blind, placebo-controlled, 12-week study. Diabetes Obes Metab. 2013;15:1136-45.

12. Inagaki $\mathrm{N}$, Kondo $\mathrm{K}$, Yoshinari $\mathrm{T}$, Takahashi $\mathrm{N}$, Susuta Y, Kuki H. Efficacy and safety of canagliflozin monotherapy in Japanese patients with type 2 diabetes inadequately controlled with diet and exercise: a 24-week, randomized, double-blind, placebo-controlled. Phase III study. Expert Opin Pharmacother. 2014;15:1501-15.

13. Inagaki N, Kondo K, Yoshinari T, Kuki H. Efficacy and safety of canagliflozin alone or as add-on to other oral antihyperglycemic drugs in Japanese patients with type 2 diabetes: a 52-week open-label study. J Diabetes Investig. 2015;6:210-8.

14. Inagaki $\mathrm{N}$, Kondo $\mathrm{K}$, Yoshinari $\mathrm{T}$, et al. Pharmacokinetic and pharmacodynamic profiles of canagliflozin in Japanese patients with type 2 diabetes mellitus and moderate renal impairment. Clin Drug Investig. 2014;34:731-42.

15. Nomura S, Sakamaki S, Hongu M, et al. Discovery of canagliflozin, a novel C-glucoside with thiophene ring, as sodium-dependent glucose cotransporter 2 inhibitor for the treatment of type 2 diabetes mellitus. J Med Chem. 2010;53:6355-60.

16. Oguma T, Kuriyama C, Nakayama K, et al. The effect of combined treatment with canagliflozin and teneligliptin on glucose intolerance in Zucker diabetic fatty rats. J Pharmacol Sci. 2015;127:456-61.

17. Kuriyama C, Xu JZ, Lee SP, et al. Analysis of the effect of canagliflozin on renal glucose reabsorption and progression of hyperglycemia in zucker diabetic Fatty rats. J Pharmacol Exp Ther. 2014;351:423-31.

18. Devineni D, Morrow L, Hompesch $\mathrm{M}$, et al. Canagliflozin improves glycaemic control over 28 days in subjects with type 2 diabetes not optimally controlled on insulin. Diabetes Obes Metab. 2012;14:539-45.

19. Devineni D, Vaccaro N, Polidori D, Stieltjes H, Wajs E. Single- and multiple-dose pharmacokinetics and pharmacodynamics of canagliflozin, a selective inhibitor of sodium glucose co-transporter 2, in healthy participants. Int J Clin Pharmacol Ther. 2015;53:129-38.

20. Devineni D, Curtin CR, Polidori D, et al. Pharmacokinetics and pharmacodynamics of canagliflozin, a sodium glucose co-transporter 2 inhibitor, in subjects with type 2 diabetes mellitus. J Clin Pharmacol. 2013;53:601-10.

21. Polidori D, Sha S, Ghosh A, Plum-Mörschel L, Heise $\mathrm{T}$, Rothenberg P. Validation of a novel method for determining the renal threshold for glucose excretion in untreated and canagliflozin-treated subjects with type 2 diabetes mellitus. J Clin Endocrinol Metab. 2013;98:E867-71.

22. Sha S, Devineni D, Ghosh A, et al. Pharmacodynamic effects of canagliflozin, a sodium glucose co-transporter 2 inhibitor, from a randomized study in patients with type 2 diabetes. PLoS One. 2014;9:e110069.

23. Seino Y, Nanjo K, Tajima N, et al. Report of the committee on the classification and diagnostic criteria of diabetes mellitus. J Diabetes Investig. 2010;1:212-28.

24. Sha S, Polidori D, Farrell K, et al. Pharmacodynamic differences between canagliflozin and dapagliflozin: results of a randomized, double-blind, crossover study. Diabetes Obes Metab. 2015;17:188-97. 
25. U.S. Food and Drug Administration. FDA Drug Safety Communication: FDA warns that SGLT2 inhibitors for diabetes may result in a serious condition of too much acid in the blood. 2015. http://www.fda.gov/Drugs/DrugSafety/ucm 446845. htm. Accessed Aug 2, 2015.

26. European Medicines Agency. SGLT2 inhibitors. 2015. http://www.ema.europa.eu/ema/index.
jsp?curl=pages/medicines/human/referrals/SGLT2_ inhibitors/human_referral_prac_000052.jsp\&mid= WC0b01ac05805c516f. Accessed Aug 2, 2015.

27. Rennke HG, Denker BM. Renal pathophysiology: the essentials. 4th ed. Philadelphia: Wolters Kluwer/Lippincott Williams \& Wilkins; 2014. p. 31-64, p. 95-119. 